\title{
Ultrasonic testing of the application of the concrete defects
}

\author{
Yang Yan \\ Department of Logistics management \\ China Maritime Police Academy \\ Ningbo ,China \\ E-mail:594yangyan@sina.com
}

\begin{abstract}
This article explains the characteristics of ultrasonic detection in concrete and its concrete defects detection, the basic principles of ultrasound in raises for concrete imperfect area and a hole in the process of test method and testing for the matters of attention.
\end{abstract}

Keywords-Ultrasonic; concrete; defects

\section{INTRODUCTION}

In all the requirements of building, safety is first. And for existing repair and maintenance of the old buildings will cost a lot of money, concrete as the most widely used in building materials, the largest use a kind of material, concrete application of ultrasonic inspection technology can make maintenance greatly reduce blindness, which can greatly reduce the spending. Concrete ultrasonic inspection technology help to evaluate the stability of the new and old buildings and integrity, capable of whole or part of the new and old buildings quality status monitoring, can be used to evaluate building materials and structure of the nature and performance.

\section{II . CONCRETE ULTRASONIC TESTING OF CHARACTERISTICS}

Concrete is made of cementing material, fine aggregate and coarse aggregate and admixture, through certain proportion coordination, and the mixing, transportation, water, and vibrating; the curing process to make a series of artificial stone material. Due to its by the solid-liquid-gas three-phase is composed of the sticky of plastic composite material, its exist a distribution is extremely complex interface, so in the propagation of the ultrasonic concrete situation than in even more complex medium, mainly for:

\section{A. Use only low-frequency ultrasonic wave}

Because there are hereby widely concrete characteristic impedance of big differences of medium interface, ultrasonic wave propagation process, in these interface happen scattering is very clear, especially high frequency component scattering is more serious. So, in order to make the ultrasonic wave propagation distance in concrete are

larger, typically use the $20-300 \mathrm{kHz}$ low-frequency ultrasonic wave.

\section{B. Launch of the ultrasonic directionality poor}

Due to the low frequency ultrasound, in the spread of the concrete in a long wavelength, and launch the diameter of the transducer is small. Ultrasound in concrete many of the interface of the irregular produces reflection and refraction, and mutual interference and stack, make most of the sound waves generate diffusion.

\section{Ultrasound in concrete is not linear transmission}

Because of the concrete and qualitative, ultrasound in countless irregular stones and mortar happening on the interface reflection and refraction, make receives the sound waves along the test is not a straight line direction spread.

\section{Received signals is very complex}

Ultrasonic wave in the concrete due to spread, can produce many times along the way of surface wave and refraction wave and the wave pattern conversion produce shear waves of these many complex wave with different phase, different paths are united, make its spread to receive the signal of the transducer is very complex. Use of ultrasonic receiving signal changes can be strength of concrete and defects comprehensive evaluation.

\section{THE ULTRASONIC CONCRETE DEFECTS BASIC PRINCIPLE}

At present, in civil engineering, the defects of concrete application of ultrasonic testing technology than in other industrial department that ordinary, produce there are many reasons for this phenomenon, the main reason is testing environment is too complicated. Modern building and bridge, locks and other buildings the main material of reinforced concrete is uniform, porous, changeful with the opposite sex material, it not only has a complex structure, and the properties of the great dispersion.

Concrete defects of ultrasonic detection technology compared with metal ultrasonic flaw detection technology, the research and application of the late start, the speed of development also slower, and the technical difficulties. Because the metal materials relative to the wavelength of the ultrasonic it is homogeneous material and the same type metal materials the velocity of basic fixed, so metal detection room with high frequency (frequency transducers for 1-10 MHz) ultrasonic pulse wave to reflection wave characteristic parameters as the basic foundation of discriminating defects, before the first test instrument with standard specimens calibration is good, the test, according to the signal features value judgment directly reflect the size 
and location of the defects. And concrete with inhomogeneous play the sticky plastic materials, ultrasonic pulse wave absorption and scattering attenuation is bigger, which are more likely to high frequency component attenuation, and according to the quality of the materials and dosage is different, the strength of concrete and the change of the moisture content, concrete velocity in considerable scope change, can't precalibrated or set a discriminating defects of the index.

Used for concrete defects detection of ultrasonic refers to the waveform display function with the ultrasonic detector and frequency of the non-metallic $20-30 \mathrm{kHz}$ ultrasonic transducer, measurement of ultrasonic pulse wave propagation speed in concrete (hereinafter referred to as the sound velocity), the first wave of amplitude (hereinafter referred to as volatility) and accept the signal frequency (hereinafter referred to as the main frequency) acoustic parameters, and according to these parameters and the relative change, determine the defects of concrete. Use of ultrasonic testing the basic principle of concrete defects as follows.

A.Ultrasound in concrete to spread, meet than its wavelength of small size defects will produce the diffraction, so that the acoustic distance spread increases, prolonged. According to the changes of sound or sound velocity, discrimination and calculation of the size of the defects.

$B$. Ultrasound in concrete to spread, meet honeycomb, hole, crack defects, a majority of pulse wave will in the defect interface for scattering and reflection, to receive the acoustic energy transducer (amplitude) significantly reduce, can according to the degree of change in volatility discriminating defects of the nature and size.

C. Each frequency components of the pulse wave attenuation extent in the defect interface is different, the higher the frequency of the pulse wave, the greater the attenuation, so ultrasonic pulse wave through the defective concrete, received signals the frequency is decreased obviously. But according to receive the signal frequency or the Lord frequency spectrum change, discriminating defects breakdown.

$D$. Ultrasonic through the defect, part of the pulse wave or several times by diffraction reflected by path and phase change, different path or different phase ultrasonic stacked, cause received signal waveform distortion, may refer to the distortion waveform analysis judgment of concrete defects.

When the composition of concrete material, the technology conditions, internal quality and test the same in distance, the point sound velocity, amplitude and frequency of the acoustic parameters are generally no significant difference. If a part of existing concrete hole, imperfect or crack defects such as, and destroy the concrete integrity, through the place with the normal conditions of ultrasonic concrete compare, sound obvious during slightly long (sound velocity reduce), amplitude and frequency is reduced and the waveform also have a serious distortion, can according to the sound of the parameters of the relative changes to judge the nature of the defects of concrete, size and scope.

\section{Ultrasonic tesTING OF THE BASIC METHODS OF CONCRETE DEFECTS}

Concrete defects, it is to point to destroy the continuity of the concrete and integrity, and to a certain extent, reduce the strength and durability of the concrete of the imperfect area, hole, crack or mingled with sediment, and sundry etc. The so-called imperfect area, it is to point to concrete for leakage vibration, segregation or stones, in the form of honeycomb overhead, or by the lack of cement and the formation of the SongSanZhuang, or accidental injury caused by the loose shape area.

The ultrasonic detection concrete defects, general is according to the structure of the components or geometric shape, the environment, size and can provide the test surface conditions, choose different test method. Here introduced special concrete defect imperfect area and hole detection, concrete internal defects range can't intuitive judgment, generally measured structure according to the actual appropriate choice of test method.

\section{A. The method of two parallel test}

Can provide for two parallel to the surface and section size and not so big component, generally USES the method of testing. Test, first the T/R transducer are set including a couple parallel tests on the corresponding point, point by point when the sound, amplitude and read the frequency value. When some point data is abnormal, must clean surface reeated measure, if reiteration data still unusual, it should be abnormal around in section measuring range, in the other pair parallel test surface decorate ultrasound point respectively, $\mathrm{T} / \mathrm{R}$ respectively in measuring transducer coupling corresponding point by point on testing to determine the location of the defects and scope. If the first pair on the test data of test no abnormalities are found, general can no longer for the detection of the second test. And, of course, is to prevent undetection also can be in the other pair test surface decorate point by point by point detection on row, but each row in measuring section position best and the first pair in the test point section stagger.

The method of simple save trouble, as long as the corresponding point coordinate position established, two direction can be measured based on sound, after the changes of the volatility of the discriminating defects space, accuracy is higher, it is the most commonly used test methods.

\section{B. The method of A pair of parallel test}

For can only provide a parallel surface of components can adopt bevel measurement method for testing. Testing procedures and the method of same, it is to be in commonly the basis of measuring point for measuring around 
suspicious inclined (including horizontal and vertical direction of the inclined measure), to determine the location of defects. Test, suspected in the area around the parallel two surface corresponding point to decorate to measure. When the suspicious of data, then around the suspicious data range the inclined.

Not too big to high beams of the kinds of members, T/R along the horizontal displacement transducer layout for the inclined, first on T1-R1 to T7-to measure R7, assuming T4R4 and T5-R5 two abnormal data, namely the horizontal direction will $\mathrm{T} / \mathrm{R}$ transducer stagger $2-3$ points, shifted $\mathrm{T}$ (1)-R (1) to (5) T-R (5) on the inclined, if the T (2)-R (2), T (3)-R (3) two appear abnormal data, it can coordinate geometry, locate the defects by roughly range. If need further judge exactly where defects, encryption on the fine points or turn on the direction of inclined.

With a certain height to the structure or component, but will $\mathrm{T} / \mathrm{R}$ along the vertical displacement transducer layout for inclined measure, first on T1-R1 to T6-R6 for test, and then in the vertical direction will T/R transducer stagger 2-3 points, shifted T (1)-R (1) to (5) T-R (5) on the inclined. When components or structure section size is bigger, also can adopt the vertical and horizontal inclined inclined measuring method of combining the measure on fine, in order to achieve the purpose of defect position accuracy. Generally speaking, from two directions inclined to measure, the determination of the defect position and scope, more accurately.

\section{V . RESEARCH SIGNIFICANCE}

Quality of concrete construction project of ultrasonic detection technology is important in the fields of nondestructive testing technology. Use ultrasound method to detect the defects of concrete, is a kind of effective method, but in some needs to be further improvement and development, such as detection method still needs some improvement, data acquisition accuracy to be raised, detection instrument of acoustic parameters should exist. It can be said that with the ultrasonic method of concrete material defects detection is a kind of very has the potential to test means, with broad space for development, it needs a lot of scientific workers to constantly perfected and innovation, in order to better serve the engineering business.

\section{REFERENCES}

[1]Technical specification for inspection of concrete defects by ultrasonic method CECS 21:2000 [J]. Shanxi building scientific research institute. Shanghai tongji university.2000.

[2]Technical specification for testing concrete strength by ultrasonicrebound combined method CECS 02:88 [J]. China building scientific research institute. 2000

[3] Zhang zhi ping, Qiu ping. Ultrasound in concrete quality testing of application [M]. Chemical industry press. 2006, 5-

[4] Liu Qi Wei, Chen Yue Hui etc. Ultrasonic nondestructive testing in the concrete application of defect evaluation [J]. East China highway. 2003, 8-

[5] The Chinese mechanical engineering society nondestructive testing to learn. Overview of nondestructive testing [M]. Mechanical industry press. 1993. 